\title{
Digital Tomosynthesis Mammography
}

National Cancer Institute

\section{Source}

National Cancer Institute. Digital Tomosynthesis Mammography. NCI Thesaurus. Code C70761.

Mammography that uses multiple X-ray pictures of each breast to produce a 3dimensional rendering of the entire breast. 